\title{
Normal Magnetic Resonance Perfusion Imaging and Atypical Posterior Reversible Encephalopathy Syndrome in Chronic Kidney Disease
}

\author{
Yerim Kim, MD, MSc ${ }^{1}$, Yong Soo Shim, MD, PhD ${ }^{2}$ \\ 'Department of Neurology, Kangdong Sacred Heart Hospital, Hallym University College of Medicine, Seoul, ${ }^{2}$ Department of Neurology, Bucheon St. Mary's \\ Hospital, College of Medicine, The Catholic University of Korea, Bucheon, Korea
}

Background: Posterior reversible encephalopathy syndrome (PRES) is classically characterized by symmetric vasogenic edema in the parietooccipital areas, but may occur at other sites with varying imaging appearances.

Case Report: A 55-year old female with chronic kidney disease (CKD) was admitted to the emergency room, presenting with nausea, vomiting and seizure. The initial blood pressure was $145 / 90 \mathrm{mmHg}$. Fluid attenuated inversion recovery demonstrated diffuse vasogenic edema in the bilateral cortical and subcortical white matters involving the frontal lobes. Perfusion magnetic resonance imaging (MRP) showed no hyper- or hypoperfusion at blood pressure levels of 140/50 mmHg. A follow-up magnetic resonance imaging at 3 weeks later demonstrated complete resolution of previous lesions.

Conclusions: Earlier reports have demonstrated that PRES can occur in cases of atypical distributions, and features of imaging findings and normotensive settings. It is important to note that PRES is a dynamic process. As a result, we suggest that MRP must be considered in the appropriate temporal framework, to avoid misinterpretation of the other diseases, especially in CKD patients.

\author{
Received January 17, 2017 \\ Revised March 14, 2017 \\ Accepted April 1, 2017 \\ Corresponding Author: \\ Yong Soo Shim, MD, PhD \\ Department of Neurology, Bucheon St. \\ Mary's Hospital, College of Medicine, \\ The Catholic University of Korea, 327 \\ Sosa-ro, Wonmi-gu, Bucheon 14647, \\ Korea \\ Tel: $+82-32-340-2020$ \\ Fax: +82-32-340-7227 \\ E-mail: ysshim@catholic.ac.kr
}

Copyright $\odot 2017$ The Korean Neurocritical Care Society

J Neurocrit Care 2017;10(1):41-45

Key words: Posterior reversible encephalopathy syndrome; Brain magnetic resonance imaging; Brain Perfusion; Chronic Kidney Disease

\section{INTRODUCTION}

Posterior reversible encephalopathy syndrome (PRES) is classically characterized by symmetric vasogenic edema in the parietooccipital areas, but may occur at other sites with varying imaging appearances. ${ }^{1,2}$ More than three fourths of patients with PRES are hypertensive, while some subjects have normal or only mildly elevated blood pressure., ${ }^{2,3}$ The exact patho-mechanism in PRES has not been elucidated.
However, in order to avoid the misinterpretation of atypical PRES as any the other disease, awareness of these atypical patterns and situations are important.

\section{CASE REPORT}

A 55-year old female was admitted to the emergency room on 25 November 2015, presenting with nausea and

(c) This is an Open Access article distributed under the terms of the Creative Commons Attribution Non-Commercial License (http://creativecommons.org/licenses/by$\mathrm{nc} / 4.0$ ) which permits unrestricted non-commercial use, distribution, and reproduction in any medium, provided the original work is properly cited. 

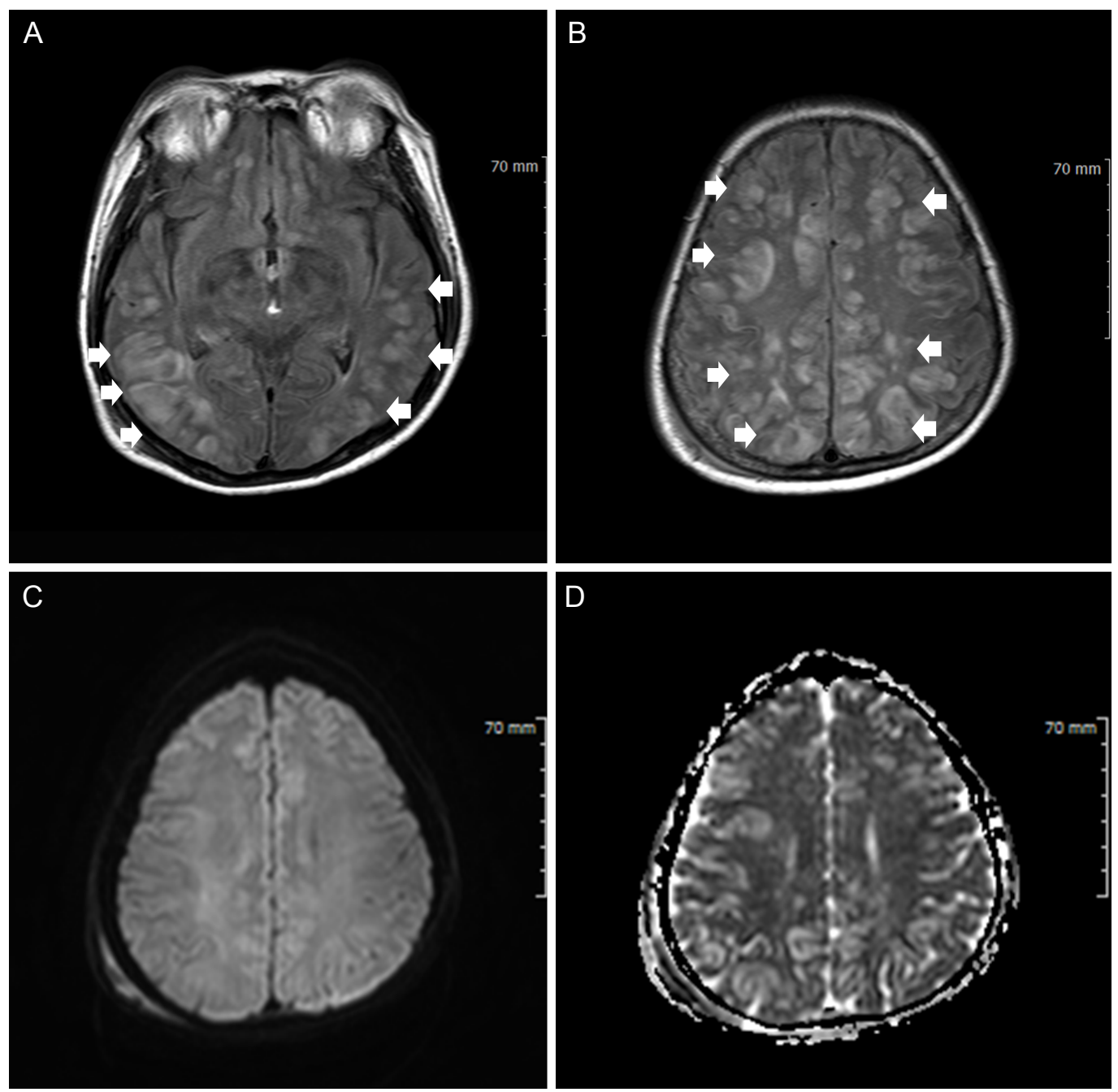

Figure 1. Initial brain-magnetic resonance imaging. (A, B) FLAIR demonstrated diffuse vasogenic edema in the bilateral cortical and subcortical white matters involving the frontal lobes (arrows). (C) DWI revealed non-significant high signal. (D) ADC map demonstrated high signal intensities in these regions. FLAIR, fluid attenuated inversion recovery; DWI, diffusion-weighted imaging; ADC, apparent diffusion coefficient.

vomiting. She had a 1-day history of drowsiness and vomiting. Her past medical history included hypertension, diabetes, hyperthyroidism and chronic kidney disease (CKD) without renal replacement therapy. Prior medications included cilostazol, carvedilol, gliclazide, linagliptin and methimazole. Her initial blood pressure was 145/90 mmHg. Laboratory findings were as follows: hemoglobin, $14.9 \mathrm{~g} / \mathrm{dL}$; total white cell count, $12.72 \times 10^{9} / \mathrm{L}$; platelet count, $145 \times 10^{9} / \mathrm{L}$; serum creatinine, $2.1 \mathrm{mg} / \mathrm{dL}$; and blood urea, $49.2 \mathrm{mg} / \mathrm{dL}$. Initial levels of the thyroid hormone were normal. Fundoscopic examination revealed hypertensive retinopathy without papilledema.
The patient developed seizures after admission. Intravenous lorazepam $4 \mathrm{mg}$ was given for acute control of seizure, after which she underwent brain magnetic resonance imaging (MRI). Fluid attenuated inversion recovery demonstrated diffuse vasogenic edema in the bilateral cortical and subcortical white matters involving the frontal lobes. While diffusion-weighted imaging revealed nonsignificant high signals, the apparent diffusion coefficient map demonstrated high signal intensities in these regions (Fig. 1A-D). There was no history of cytotoxic immunosuppressive therapy. Perfusion MRI (MRP) showed no hyperor hypoperfusion at blood pressure levels of $140 / 50 \mathrm{mmHg}$ 

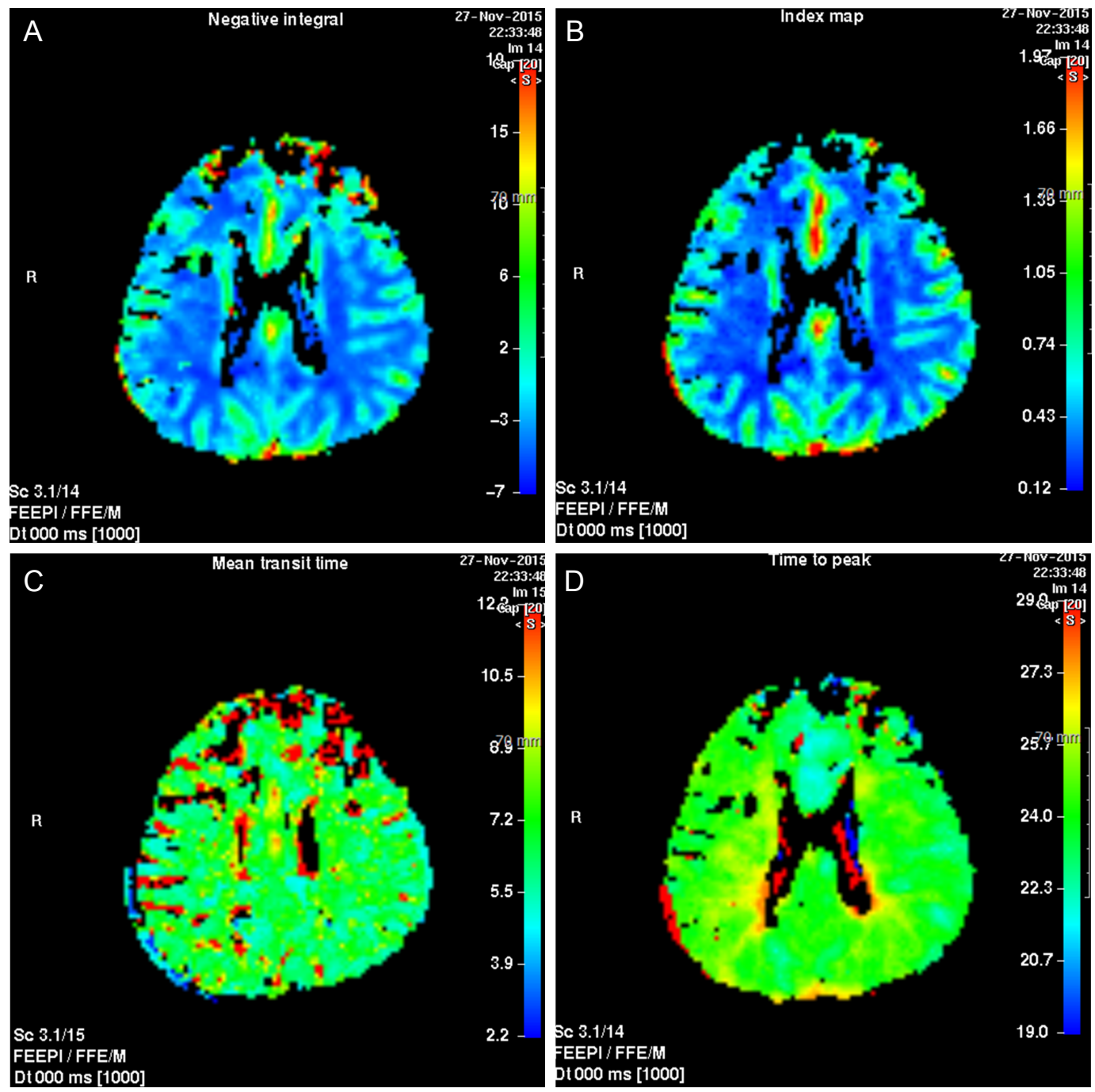

Figure 2. Normal perfusion MRI at blood pressure levels of $140 / 50 \mathrm{mmHg}$. (A) Cerebral blood volume, (B) cerebral blood flow, (C) mean transit time, (D) time to peak. MRI, magnetic resonance imaging.

(Fig. 2A-D). After close monitoring of blood pressure, her symptoms were resolved. Considering low recurrence rate of seizure, we did not prescribe any antiepileptic drugs. The 3 weeks follow-up MRI demonstrated complete resolution of previous lesions (Fig. 3A-B). This might be interpreted as atypical PRES

\section{DISCUSSION}

PRES is a neurologic syndrome, diagnosed by clinical and radiologic features. The typical presentation includes headache, mental change, visual symptoms, and seizures. The characteristic MRI findings are consistent with vasogenic edema predominantly localized to the posterior hemisphere. It is not entirely elucidated why PRES favors the posterior circulation, but this might be related to a relative lack of sympathetic innervation at the level of the arterioles supplied by the vertebrobasilar system, compared with the anterior circulation. ${ }^{4}$ However, PRES is not a posterior regional phenomenon. Additionally, more than $70 \%$ of patients with PRES are hypertensive, though significant proportions have normal or only mildly raised blood pressure. ${ }^{5}$ Previous reports have demonstrated that PRES can occur in cases of atypical distributions, and features of imaging findings and normotensive settings. ${ }^{4,6}$ The exact patho- 

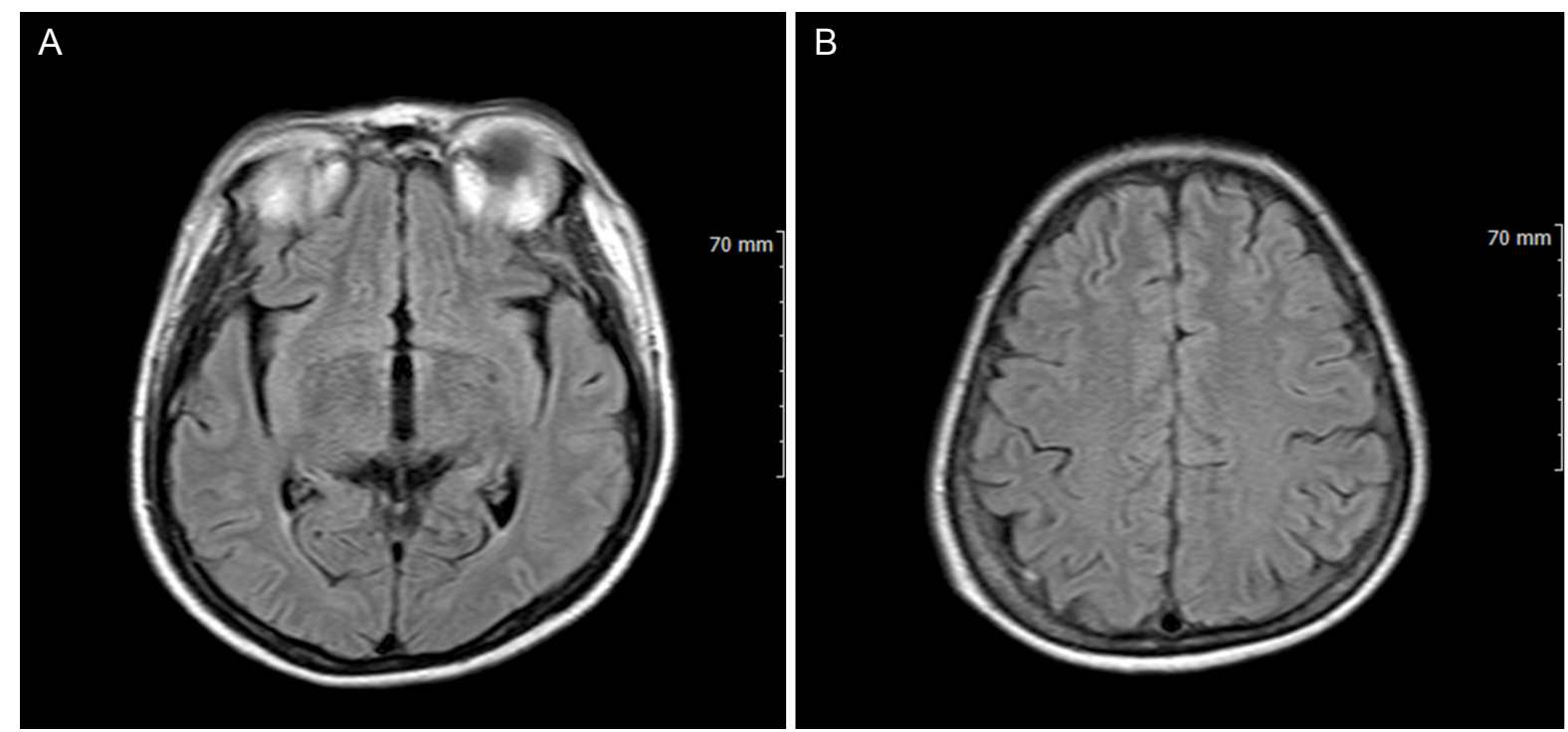

Figure 3. FLAIR after 3 weeks. The follow-up magnetic resonance imaging demonstrated complete resolution of previous lesions. FLAIR, fluid attenuated inversion recovery.

physiology of PRES remains controversial. One currently favored theory is hypertension with autoregulatory failure. When the upper limits of autoregulation are exceeded, there is a development of blood vessel alteration, capillary bed injury, vasogenic edema, and hyperperfusion. However, it does not explain why PRES occurs in the absence of hypertension. ${ }^{5}$ Furthermore, although this theory suggests cerebral hyperperfusion, some evidences demonstrate cerebral hypoperfusion. ${ }^{7}$ Another earlier theory is associated with a systemic inflammatory process including immune system activation, endothelial injury, vasoconstriction and hypoperfusion. However, it is not clear why some cases of PRES occur without any inflammatory state. ${ }^{5}$ Despite that, one large series of cases demonstrated that atypical distribution of PRES have a higher incidence than commonly perceived. PRES is not an entirely posterior phenomenon, but rather shows in a gradient-like pattern, reflecting the gradient of sympathetic innervations. ${ }^{4}$

In our case, vasogenic edema occurred at relatively not very high blood pressure, and the imaging findings of MRI showed atypical appearances. Based on a previous report, although peak systolic blood pressure is usually between $170-190 \mathrm{mmHg}, 10-30 \%$ of patients have normal or mildly elevated blood pressure. In clinical settings, uremia in CKD patients may mediate their effect on the vascular endothelium or other regions of vasogenic control. Furthermore, it is important to note that PRES is a dynamic process. The MRP in PRES is significantly increased in the acute hypertensive setting, but after the blood pressure has reduced, perfusion also tends to decrease. ${ }^{8}$ Because contrast perfusion method was contraindicated in CKD patients, the exact perfusion mechanisms in these patients were not elucidated. However, because the MRI (including perfusion) was performed on this patient to rule out hyperacute ischemic stroke, this case might propose some critical information for the hemodynamic mechanisms of atypical PRES.

In conclusion, we suggest that MRP should be considered in the appropriate temporal framework, to avoid misinterpretation of other diseases, such as acute cerebral ischemia, cerebral venous thrombosis, encephalitis, and brain tumor, especially in patients with CKD.

\section{REFERENCES}

1. Fugate JE, Claassen DO, Cloft HJ, Kallmes DF, Kozak OS, Rabinstein AA. Posterior reversible encephalopathy syn- 
drome: associated clinical and radiologic findings. Mayo Clin Proc 2010;85:427-32.

2. Bartynski WS. Posterior reversible encephalopathy syndrome, part 1: fundamental imaging and clinical features. AJNR Am J Neuroradio/ 2008;29:1036-42.

3. Hobson EV, Craven I, Blank SC. Posterior reversible encephalopathy syndrome: a truly treatable neurologic illness. Perit Dial Int 2012;32:590-4.

4. McKinney AM, Short J, Truwit CL, McKinney ZJ, Kozak OS, SantaCruz KS, et al. Posterior reversible encephalopathy syndrome: incidence of atypical regions of involvement and imaging findings. AJR Am J Roentgenol 2007;189:904-12.
5. Bartynski WS. Posterior reversible encephalopathy syndrome, part 2: controversies surrounding pathophysiology of vasogenic edema. AJNR Am / Neuroradio/ 2008;29:1043-9.

6. Stevens C), Heran MK. The many faces of posterior reversible encephalopathy syndrome. Br J Radio/ 2012;85:1566-75.

7. Bartynski WS, Boardman JF. Catheter angiography, MR angiography, and MR perfusion in posterior reversible encephalopathy syndrome. A/NR Am / Neuroradiol 2008;29:447-55.

8. Schwartz R, Mulkern R, Vajapeyam S, Kacher DF. Catheter angiography, MR angiography, and MR perfusion in posterior reversible encephalopathy syndrome. A/NR Am / Neuroradiol 2009;30:E19. 